\title{
Museu virtual: um novo olhar para a informação e comunicação na museologia
}

\section{Robson da Silva Teixeira}

\author{
Mestrando em Desenvolvimento Local, UNISUAM, \\ Rio de Janeiro. Bibliotecário-chefe da Biblioteca \\ do Instituto de Física/UFRJ \\ Rio de Janeiro.
}

http://dx.doi.org/10.1590/1981-5344/1908

Este artigo aborda os espaços virtuais de museus, a partir da questão de uma representação virtual do Instituto de Física da Universidade Federal do Rio de Janeiro (UFRJ); esse museu virtual contempla coleções de ciência e tecnologia do Instituto de Física (IF) como subsídio para a pesquisa museológica e para a construção da história e memória do ensino de física no Brasil. Este artigo está voltado para a memória institucional do IF (UFRJ), que em 2013 fez 49 anos, e ocupa um lugar de destaque entre as instituições de ensino e pesquisa.

Palavras-chave: Tecnologia da Informação; museu virtual; organização da memória científica; Universidade Federal do Rio de Janeiro (UFRJ).

\section{Virtual museum: a new look for information and communication in museology}

This article discusses the virtual spaces of museums, from the question of a virtual representation of the Physics Institute of the Federal University of Rio de Janeiro (UFRJ), that includes virtual museum collections science and technology of the Institute of Physics (IF) as a subsidy museum for research and the construction of history and memory of physics education in Brazil. This article is focused on the institutional memory of the IF (UFRJ), who did 49 years in 2013, and occupies a 
prominent place among the institutions of education and research.

Keywords: Information Technology; virtual museum; scientific memory organization.

Recebido em 29.10.2013 Aceito em 02.10.2014

\section{Introdução}

Há um enorme campo de trabalho a ser explorado para a organização da memória institucional das universidades públicas brasileiras. Evidentemente, compete àquelas instituições, produtoras e difusoras do conhecimento, zelar por esses documentos, já que eles são imprescindíveis para a construção, conhecimento e desenvolvimento da História e da Pesquisa no país. Nesse sentido, torna-se necessário preservar digitalmente a memória institucional a partir de um museu virtual que permita o acesso à biografia docente, documentos administrativos, relatórios de pesquisa, mobiliário, fotografias e instrumentos científicos, com o objetivo de salvaguardar a memória do Instituto de Física (IF) da Universidade Federal do Rio de Janeiro (UFRJ). O museu virtual não existe no mundo real/físico, mas existe a coleção de documentos diversificados e para a Museologia esses documentos são considerados objetos musealizados, por isso fez-se uma pesquisa para coletá-los e denominá-los objetos museológicos. É um estudo preliminar focado na memória institucional do curso de Física da Universidade Federal do Rio de Janeiro (UFRJ). É na perspectiva de facilitar o acesso à informação científica e tecnológica na área de física que se insere o presente estudo, que visou à constituição de um museu virtual, que permita a busca de todo um conjunto de conteúdos digitais originários da pesquisa e do ensino gerados pela comunidade científica do Instituto de Física da UFRJ.

Pelo exposto, o presente artigo teve como objetivo elaborar o Museu Virtual do Instituto de Física da Universidade Federal do Rio de Janeiro (UFRJ), conforme o modelo de Museu Virtual de Composição Mista, tendo como acervo o material produzido e usado pelos pesquisadores, visando por meio de processo de digitalização trazer à público a história da pesquisa praticada pelos professores brasileiros no contexto nacional e internacional. Dentro desta perspectiva, delimitou-se três objetivos específicos: pesquisar a produção científica dos professores/fundadores do IF - artigos no Brasil e no exterior; identificar e descrever as coleções que farão parte do museu virtual; e gerar um arquivo de depoimentos orais oriundo da pesquisa com professores eméritos. 


\section{Organização da memória científica gerada nas universidades públicas brasileiras: um estudo de caso no Instituto de Física da Universidade Federal do Rio de Janeiro (UFRJ)}

Segundo o site UFRJ história (2012), o Instituto de Física da Universidade Federal do Rio de Janeiro (IF/UFRJ) foi criado em 19 de março de 1964 e faz parte do Centro de Ciências Matemáticas e da Natureza (CCMN) da referida universidade. Antes da criação do Instituto, o curso de física fazia parte da Faculdade Nacional de Filosofia (FNFI) e reunia cinco cátedras de Física, á com vistas a sua finalidade de formar Bacharéis e Professores Licenciados. O Instituto de Física (IF) foi criado por ocasião da reforma universitária, que reuniu os cursos de física, então existentes, em escolas e faculdades do Rio de Janeiro pertencentes à Universidade do Brasil (UB). O IF buscava constituir-se com a contratação de professores necessários às atividades de ensino, e procurava quadros superiores para a implantação de atividades de pesquisa e a preparação para a pós-graduação, que, até então, não existia no Instituto.

Baseado no livro Instituto de Física-UFRJ 45 anos (2010, p.12), em 1966 o Instituto instalou-se no Fundão (Ilha do Governador, Rio de Janeiro) efetivamente, contava com pouco mais de 10 professores, dentre eles Cesar Lattes, José Leite Lopes, Jayme Tiomno e Plínio Sussekind Rocha. A graduação era dividida em dois ciclos: Física Básica e Física Avançada; e a licenciatura foi criada em 1969. O mestrado em física foi implantado em 1977 e o doutorado instaurado em 1979.

\subsection{Biografia docente: Professores/Fundadores do Instituto de Física da UFRJ}

O livro Instituto de física (2010, p.10) relata que os pesquisadores que fizeram parte da antiga Faculdade Nacional de Filosofia (FNFI) que posteriormente se tornou o Instituto de Física (IF) são:

- Cesar Lattes: participação ativa na equipe que descobriu o méson pi. O diretor da equipe de Bristol, Cecil Frank Powell, foi laureado com Prêmio Nobel de Física em 1950 por essa descoberta. Consolidação do Laboratório de Chicaltaya, na Bolívia em 1950. Pesquisador renomado, que deu nome ao currículo Lattes ${ }^{\prime}$; o impacto do trabalho científico de Cesar Lattes levou à criação em 1949 do Centro Brasileiro de Pesquisas Físicas (CBPF). Por sua relevância no cenário científico, o Senado Federal criou na FNFI uma nova cátedra: Física Nuclear, oferecida a Cesar Lattes. Tem seis livros

\footnotetext{
${ }^{1}$ Curriculum lattes é um currículo elaborado nos padrões da plataforma Lattes, do CNPq (Conselho Nacional de Desenvolvimento Científico e Tecnológico). A Plataforma Lattes representa a experiência do CNPq na integração de bases de dados de currículos, de grupos de pesquisa e de instituições em um único sistema. Wikipédia. Disponível em: <http://pt.wikipedia.org/wiki/Espectr\%C3\%B4metro>. Acesso em: 27.08.2012.
} 
publicados, seis artigos científicos, e apresentou no Interamerican Seminar on Cosmic Rays, em 1970, um dos primeiros trabalhos sobre a descoberta da produção de mésons pi em aceleradores.

- José Leite Lopes: articulou para a criação da Comissão Nacional de Energia Nuclear (CNEN) e o Conselho Nacional de Desenvolvimento Científico e Tecnológico (CNPq). Tem publicado 58 livros, dois artigos científicos e desenvolveu sua tese sobre alta energia de espalhamento de nêutrons e prótons na Princeton University em 1945, sendo considerado um dos primeiros estudos sobre o assunto. Participou da organização do livro Quantum mechanics: a half century later: papers of a colloquium on fifty years of quantum mechanics $e$ traduziu junto com Jayme Tiomno, duas edições do livro Física na escola secundária de 1958 e 1962.

- Joaquim da Costa Ribeiro: foi o primeiro delegado do Brasil junto ao Comitê Consultivo das Nações Unidas para as Aplicações Pacíficas da Energia Nuclear. Catedrático de Física Geral e Experimental, trabalhava em colaboração com Bernardo Gross (do Instituto Nacional de Tecnologia) sobre o efeito termodielétrico (chamado Efeito Costa Ribeiro), descoberto por ele em 1944. Tem dois livros publicados, seis artigos científicos e apresentou trabalhos relevantes em eventos científicos de destaque no cenário científico, tais como: Metrologia: métodos ópticos nas medidas de comprimento de alta precisão (Conferência realizada no Instituto Nacional de Tecnologia em 13 de agosto de 1936), e Novas pesquisas tecnológicas em física (simpósio organizado pela Academia Brasileira de Ciências e o Centro de Cooperação Científica para America Latina (UNESCO) e o Conselho Nacional de Pesquisas do Brasil em 1952). Ressalta-se que a Biblioteca da Universidade de Campinas (Unicamp) absorveu todo o acervo pessoal do pesquisador Joaquim da Costa Ribeiro e, por isso, também será utilizada como fonte de pesquisa.

- Plínio Sussekind Rocha: participou da fundação do clube de cinema Chaplin-Club em 13 de janeiro de 1928. Incentivou à restauração do filme Limite (1931) de Mário Peixoto. A restauração inicia-se em 1958 e foi concluída em 1971. Renomado pesquisador deu nome a Biblioteca do IF e foi decano do Centro de Ciências Matemáticas e da Natureza (CCMN) da UFRJ. Tem dois livros publicados e sua tese: A mecânica de d'Alembert. Rio de Janeiro: UFRJ, 1962 está digitalizada e será disponibilizada no Museu Virtual. Participou da organização e tradução dos livros: Filosofia da ciência natural, 1981 e A ciência do século XX, 1971. 
- Jayme Tiomno: destaque na pesquisa de captura e desintegração de mésons e teoria de campos. Ao lado de César Lattes, José Leite Lopes, Joaquim da Costa Ribeiro e outros pesquisadores criaram o Centro Brasileiro de Pesquisas Físicas (CBPF). O CBPF organiza, desde 2010, o evento Jayme Tiomno School of Cosmology. Tem 20 livros publicados, 37 artigos científicos, e sua tese sobre contribuições à física das partículas elementares, 1966, contribuiu, significativamente, para as pesquisas desenvolvidas na época. Participou da organização do livro: Guide to literature of elementary particle physics, including cosmic rays, 1949.

\subsection{Professores eméritos do instituto de física da UFRJ}

Erasmo Ferreira e Fernando de Souza Barros - Ambos graduaram-se no curso de Física da antiga Faculdade Nacional de Filosofia (FNFI) e atualmente, são professores eméritos ${ }^{2}$ do Instituto de Física.

\subsection{Relatórios de pesquisa e mobiliário}

Foram consultados os relatórios de pesquisa utilizados pelos pesquisadores e a identificação de qual pesquisa eles se referem; posteriormente eles foram descritos e digitalizados.
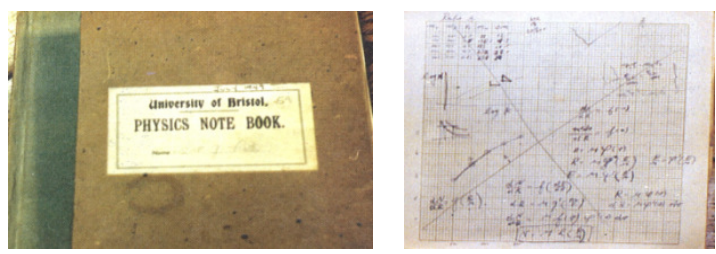

Figura 1 - caderno de laboratório que pertenceu ao pesquisador Cesar Lattes $^{3}$

Também foram pesquisados o mobiliário utilizado pelos pesquisadores do Instituto de Física, tais como: mesas, cadeiras, armários, escrivaninhas, enfim, um conjunto de móveis que faz parte da memória institucional e representa a materialização das ideias contidas na produção científica dos pesquisadores; posteriormente esse mobiliário foi descrito e fotografado. Segundo o professor Máximo Ferreira, está localizada na sala

2 Professor emérito é um título conferido por uma entidade de ensino a seus professores já aposentados, que atingiram alto grau de projeção no exercício de sua atividade acadêmica.Wikipédia. Disponível em: <http://pt.wikipedia.org/wiki/Professor em\%C3\%A9rito>. Acesso em: 27.10.2012.

3 Caderno de laboratório (note book) usado por Cesar Lattes em julho de 1947. Nele, estão os cálculos que levaram à massa do méson pi depois das exposições feitas no monte Chacaltaya, na Bolívia. Depositado na Wills Memorial Library, em Bristol. Essa descrição está no livro CESAR LATTES: a descoberta do méson e outras histórias. Rio de Janeiro: CBPF, 1999. p. 47. 
de reuniões do Instituto de Física, a mesa histórica que foi utilizada pelos professores do Departamento de Física, da antiga Faculdade Nacional de Filosofia (FNFI), na reunião na qual se decidiu que o Departamento de Física iria se tornar o Instituto de Física da UFRJ. Segundo o professor Fernando de Sousa Barros, esta mesa histórica era utilizada pelo professor Cesar Lattes ${ }^{4}$.

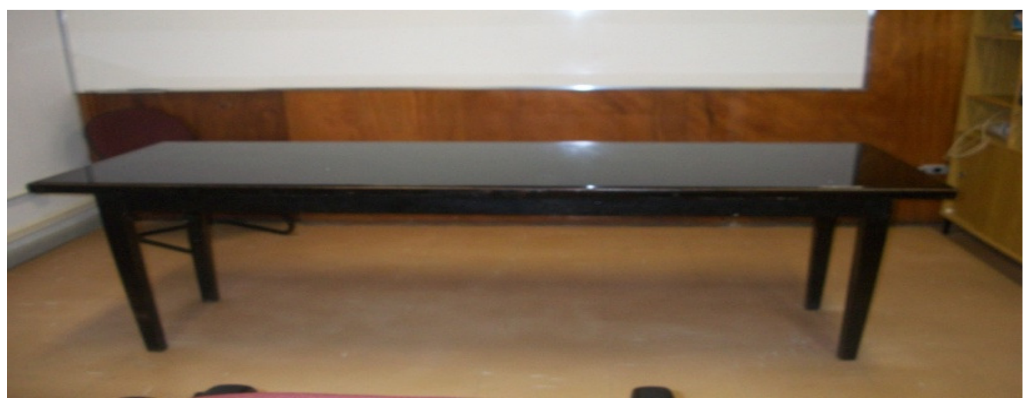

Figura 2: Mesa histórica que pertenceu ao prof. Cesar Lattes

\subsection{Documentos administrativos}

Na Biblioteca Central do Centro de Ciências Matemática e da Natureza (CCMN), encontra-se os boletins da UFRJ, oficializando a instalação do Instituto de Física 5 . Já as cartas, memorandos e atas do processo burocrático para a implantação do Instituto encontram-se em diversos departamento e na diretoria do Instituto de Física da UFRJ ${ }^{6}$. Esses documentos foram digitalizados e tratados como objetos museológicos.

\subsection{Fotografias de época}

Neste tópico foi dada ênfase especial às fotografias que comprovam o surgimento de atividades de pesquisa no IF/UFRJ, pois na época da FNFI, os professores realizavam essas atividades no CBPF. Um enfoque especial foi dado ao surgimento dos cursos de pós-graduação, quando as turmas foram de professores do próprio IF, pois uma quantidade expressiva desses professores participou da montagem dos laboratórios de pesquisa enquanto desenvolviam suas teses. O livro Instituto de física (2010, p. 47) descreve algumas fotografias localizadas:

- Foto da Instalação do Laboratório de Baixas Temperaturas com recursos do Banco Nacional de Desenvolvimento (BNDE) e a Financiadora de Estudos e Projetos (FINEP);

Depoimento oral do Professor Emérito do Departamento de Física Nuclear Fernando de Sousa Barros em 14 de setembro de 2012.

Entrevista com a Bibliotecária-chefe da Biblioteca Central do CCMN/UFRJ em 30 de agosto de 2012.

Depoimento oral da secretária da Diretoria do Instituto de Física em 15 de julho de 2012. 
- Foto da primeira turma do mestrado em física - 1977;

- Foto da primeira turma do doutorado em física - 1979;

- Foto com a primeira equipe do Laboratório Didático do Instituto de Física da UFRJ, criado em 1988;

- Foto do Laboratório Didático do Instituto de Física da UFRJ (LADIF), que é composto por três salas de experimentos (salão principal) e um laboratório de áudio-visual.

\subsection{Instrumentos científicos}

Espectrômetro ${ }^{7}$ este instrumento foi muito utilizado pelo professor Joaquim da Costa Ribeiro nas suas pesquisas na década de 1960 e segundo o livro Instituto de Física (2010, p.32) possibilitou a publicação de diversos trabalhos científicos. Esse instrumento científico pertenceu ao Instituto de Física e foi doado ao Museu de Astronomia e Ciências Afins (MAST) em $2006^{8}$.

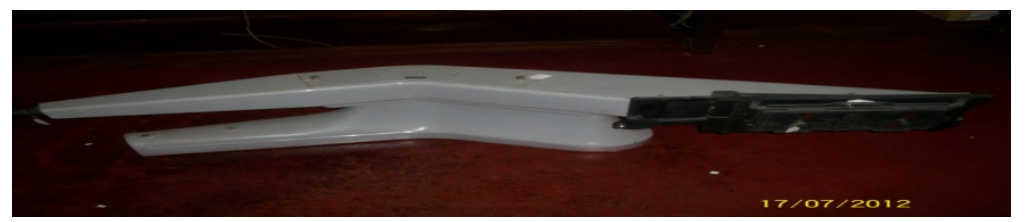

Figura 3: Espectrômetro

\section{Referencial teórico}

Para o Conselho Internacional de Museus (2012), o termo museu é entendido como sendo:

uma instituição permanente, sem fins lucrativos, a serviço da sociedade e de seu desenvolvimento, aberta ao público, que adquire, conserva, pesquisa, divulga e expõe, para fins de estudo, educação e lazer, testemunhos materiais e imateriais dos povos e seu ambiente.

Baseado em Scheiner (1998, p.89) pode-se dizer que a Teoria Museológica vem permitindo compreender "o caráter fenomênico do Museu e sua capacidade de manifestar-se de diferentes maneiras, no

\footnotetext{
7 Espectrômetro é um instrumento óptico utilizado para medir as propriedades da luz em uma determinada faixa do espectro eletromagnético, sua estrutura basicamente se resume a existência de uma rede de difração e um captador. Wikipédia. Disponível em: <http://pt.wikipedia.org/wiki/Espectr\%C3\%B4metro>. Acesso em: 27.08.2012.
}

8 UNIVERSIDADE FEDERAL DO RIO DE JANEIRO. Termo de doação no. SP-IF 001/06. 
tempo e no espaço, para além das formas instituídas e/ou já reconhecidas".

Scheiner (1998, p. 141) acredita que perceber o museu como fenômeno ou acontecimento - "portanto livre, dinâmico e plural" - permite que ele deixe de ser visto "a partir de suas expressões mais óbvias (o objeto, a exposição) e de seus limites espaciais, para brilhar em novas - e inusitadas - dimensões, entre elas, o Museu Virtual.

Andrews (1998, p.19) definiu o termo 'museu virtual' foi definido como

...uma coleção logicamente relacionada de objetos digitais compostos de variados suportes que, em função de sua capacidade de proporcionar conectividade e vários pontos de acesso, possibilitaIhe transcender métodos tradicionais de comunicar e interagir com visitantes...; não há lugar ou espaço físico, seus objetos e as informações relacionadas podem ser disseminados em todo o mundo.

Com relação ao virtual, Lévy (1999, p.92) complementa relacionando-o ao ciberespaço:

Espaço de comunicação aberto pela interconexão mundial dos computadores e da memória dos computadores, explicando que neste ambiente está sediado "o conjunto dos sistemas de comunicação eletrônicos [...], que transmitem informação proveniente de fontes digitais ou destinadas à digitalização".

Em seu artigo "A legitimidade do museu virtual" (The legitimacy of the virtual museum), Schweibenz (2004, p.3) afirma que a utilidade da Internet para os museus é bem reconhecida hoje, e o seu desenvolvimento é inevitável em função da crescente digitalização do patrimônio cultural e da demanda de tornar as coleções mais acessíveis. Para o autor, o objetivo da instituição de memória é preservar este conteúdo para gerações futuras e apoiar seu uso e administração por muitas gerações.

Baseado em Lima (2009) e analisando as características teóricas e práticas configuradas por ela para designar as novas tipologias de museu virtual, acredita-se que o Museu Virtual do Instituto de Física da UFRJ será um museu virtual de composição mista - categoria $C$, isto é, não há um museu no mundo físico e a sua coleção será convertida digitalmente. A autora ressalta que o termo virtual é utilizado tanto para indicar o que se cria por meio do computador sem existir o referente no mundo físico, como também para o que existe no mundo real (mundo físico) e sofre processo de digitalização (LIMA, 2009).

Segundo a UNESCO (2004), em função das circunstâncias relativas ao processo tecnológico de produção do Museu Virtual do Instituto de Física da UFRJ e, por conseqüência sua coleção (um patrimônio 
musealizado), a designação Patrimônio Digital será também objeto de estudo, compreendendo-se como digital, as produções convertidas a partir deste recurso, isto é, reproduzidas digitalmente. Para Lima (2009) o formato digital, em termos do seu entendimento como valor de patrimônio cultural, relaciona-se ao bem simbólico resultante de mais um novo processo tecnológico surgido em tempos recentes. No caso do museu virtual, este novo modelo se insere no âmbito do Patrimônio Digital comportando a seguinte definição proposta pela UNESCO (2004, p.5):

...recursos de conhecimento ou expressão humana, seja cultural, educacional, científico e administrativo, ou abrangendo a informação técnica, legal, médica e outros tipos de informação, [que] são cada vez mais criados digitalmente, ou convertidos de sua forma analógica original à forma digital. ... Matérias digitais incluem textos, bases de dados, imagens estáticas e com movimento, áudios, gráficos, software, e páginas WEB, entre uma ampla e crescente variedade de formatos. Muitos desses materiais são de valor e significância duradouros, e por isso constituem um patrimônio que deve ser protegido e preservado para a geração atual e futura. Esse patrimônio existe em qualquer língua, parte do mundo, e em qualquer área do conhecimento e expressões humanos.

Pierre Lévy (1999) nos diz que os profissionais não estariam explorando o suficiente todo o potencial que a hipermídia tem para auxiliar no aperfeiçoamento da área museológica, sugerindo que ao invés de apenas reproduzir exposições no meio virtual, o ideal seria criar caminhos "personalizados" de acordo com as navegações, onde o público poderia dar um retorno à instituição, uma vez que estes espaços se desvinculariam das coleções materiais. Vislumbra-se aí a oportuna condição para a existência do projeto do Museu Virtual do Instituto de Física da UFRJ como um canal entre a comunidade científica e os objetos que fizeram parte da sua história e memória. Para Silveira (2009, p. 47), procuramos nos aproximar dos domínios da Museologia quando a percebemos como uma possibilidade de arranjos entre bens simbólicos, de valor patrimonial e museológico, abarcando espaços e objetos, processos comunicacionais, enquadrando formas culturais, e como fonte da memória coletiva.

Acredita-se que as considerações sobre patrimônio mostram-nos que, assim como as coleções e o acervo, os instrumentos científicos, mobiliário, documentos administrativos - tomados como "objetos patrimoniais" - podem ser referências e/ou indicadores da memória social.

Este estudo parte das considerações de que competem às universidades, produtoras e difusoras do conhecimento, zelar por sua documentação histórica. É importante conscientizar para a preservação de documentos, já que eles são imprescindíveis para a construção da História e da Memória. Partindo desta premissa, ressaltam-se as considerações de 
Le Goff (1990, p. 540) que destaca a memória como uma fonte de identidade individual e de uma dada sociedade, e como objeto de luta das forças sociais do poder, e influi na produção e manutenção de documentos na construção mnemônica. Entretanto, há que tomar a palavra "documento" no sentido mais amplo, documento escrito, ilustrado, transmitido pelo som, a imagem, ou de qualquer outra maneira.

Com este olhar Halbwachs (2006) desenvolveu um trabalho no qual - tema da memória coletiva marca uma presença constante, estabelecendo uma escala de necessidades deste grupo social a partir de sua própria avaliação, revelando a importância das representações do grupo; procurando demonstrar a institucionalização social da memória, construtora da identidade cultural do grupo, e posteriormente, desenvolvendo assim, as concepções dos quadros sociais da memória, incorporando a atuação dos grupos jurídicos, econômicos e religiosos e assinalando que a memória social possuía não apenas uma base imaterial, mas também um fundamento material.

De acordo com Levacov (1997, p.1):

A tecnologia surge como um catalisador de mudanças, particularmente importantes e pungentes para as bibliotecas, uma vez que cria novas necessidades e altera velhos paradigmas estabelecidos ao longo de muitos séculos.

O advento das novas Tecnologias de Informação e Comunicação (TICs) e as constantes mudanças nos processos e inovações tecnológicas estão fazendo com que as bibliotecas repensem o ato de produção, acesso e da informação, buscando atender as novas perspectivas de atendimento das necessidades de seus usuários, local ou remotamente. A nova ordem mundial criou novas exigências na formação dos profissionais e no gerenciamento da informação. Segundo Lambert (2000), o papel do profissional de informação "antenado" com a evolução tecnológica e as mudanças ocorridas no acesso à informação, que facilitam a vida do usuário, estará sempre baseado na utilização das novas tecnologias para atender necessidades informacionais de pesquisadores, e ainda de qualquer tipo de usuário.

Os profissionais responsáveis pela gestão das bibliotecas universitárias devem aproximar, cada vez mais, a Biblioteca, com seus serviços e produtos dos usuários que a utilizam, fazendo com que a informação chegue aos usuários de uma maneira mais rápida, prática e eficaz; e o museu virtual pode cumprir este papel de disseminador da informação. Para Robert (1990, p. 137) as bibliotecas constituem a memória de uma organização qualquer que seja a sociedade, uma coletividade, uma empresa ou uma instituição, com vistas a harmonizar seu funcionamento e gerar seu futuro. Elas existem porque há necessidade de uma memória registrada; pois uma cultura nacional vive entre o passado e futuro, ora se dirigindo ao passado e suas glórias, ora tentando avançar em direção à modernidade. A motivação para a 
elaboração deste projeto partiu da reflexão sobre a importância de construir um museu virtual para abrigar um conjunto de documentos digitais produzidos e/ou pertencentes aos pesquisadores do Instituto de Física da UFRJ. Carvalho (2009) ressalta que a literatura brasileira não registra, no entanto, estudos exploratórios mais amplos, sendo também poucos os trabalhos que relatam à implantação de museus virtuais em universidades brasileiras. A conseqüente falta de discussão do assunto no âmbito das bibliotecas universitárias pode retardar ainda mais a oferta deste tipo de serviço de informação no país.

\section{Desenhando o museu virtual: uma metodologia descritiva}

Na primeira etapa, foi feito um levantamento bibliográfico em periódicos nacionais e internacionais para quantificar e qualificar a produção científica dos pesquisadores César Lattes, José Leite Lopes, Plínio Sussekind Rocha e Jaime Tiomnio;

Na segunda etapa, foram identificados e descritos os objetos que constituiriam o Museu Virtual. Para isso, inicialmente, foi feita uma busca pelo Instituto com o objetivo de coletar mobiliário e instrumentos científicos, que posteriormente foram fotografados e/ou digitalizados, para que depois fosse feito a sua caracterização como patrimônio da Ciência e Tecnologia (C\&T) do Brasil, situando-os como uma coleção histórica de ensino e pesquisa, no âmbito das coleções universitárias. Em seguida, foi construída a trajetória da formação da coleção, através do levantamento de informações sobre os objetos que foram usados nas aulas práticas das disciplinas relacionadas à Física, e nas aulas práticas e nas pesquisas realizadas no curso de pós-graduação em Física;

Na terceira etapa, foi feito um levantamento em fontes primárias documentos dos pesquisadores, tais como: correspondências passivas e ativas, relatórios de pesquisa e caderneta de campo, e assim poder relacionar os objetos as pesquisas desenvolvidas por eles. Foi feita também uma pesquisa iconográfica, para coletar fotografias e um levantamento para coletar documentos administrativos, como boletins e atas e demais documentos textuais;

Na quarta etapa, foi gerado um arquivo de depoimentos orais, que também está acessível através da página do Museu Virtual; esse arquivo de depoimentos foi gerado a partir da aplicação de um questionário/entrevista aos pesquisadores eméritos Erasmo Ferreira e Fernando de Sousa Barros, que atualmente fazem parte do quadro de professores do IF e foram alunos de graduação e pós-graduação do Instituto. Por fim, também foi visitada a Biblioteca da Universidade de Campinas (Unicamp), que absorveu todo o acervo pessoal do pesquisador Joaquim da Costa Ribeiro, com o intuito de coletar documentos pertencentes ao pesquisador e que puderam fazer parte do acervo do Museu Virtual. Ressalta-se que como já visto anteriormente, o termo Museu Virtual é um termo consagrado na literatura científica, por este 
motivo, optou-se em utilizar, neste primeiro momento, o termo Memória Virtual como identificador do site, pois a gama de informações ficará abarcada dentro da página da Biblioteca do IF e posteriormente, o Museu Virtual ganhará uma página própria.

\section{Resultados Parciais/Finais}

Este estudo possibilitou que o Museu Virtual fosse capaz de dar acesso à biografia docente, documentos administrativos, relatórios de pesquisa, mobiliário, fotografias e instrumentos científicos, isto é, uma gama de informação científica gerada no Instituto de Física da Universidade Federal do Rio de Janeiro. É uma interface única que contempla diferentes documentos científicos e/ou históricos existente no Instituto de Física da Universidade Federal do Rio de Janeiro (UFRJ).

\section{Considerações Parciais/Finais}

O objetivo principal desta pesquisa - constituir um Museu Virtual, que permita acesso a uma gama de informação científica gerada e/ou pertencentes ao Instituto de Física da Universidade Federal do Rio de Janeiro - foi alcançado. O Museu Virtual está disponível aos usuários e pode ser localizada a partir do endereço: WWW.biblioteca.if.ufrj.br/memoriavirtual. Como reflexão para pesquisas futuras, acredita-se que há indícios fortes de que este trabalho deve ser contínuo, que o museu virtual não está totalmente pronto, precisa de ajustes, há também, a necessidade constantes de revisões e atualizações para que a interface esteja sempre em convergência com as necessidades dos usuários que a utilizam.

\section{Referências}

ANDREWS, J., SCHWEIBENZ, W.: The Kress study collection virtual museum project, a new medium for old masters. Art Documentation, v. 17 , n. 1, Spring Issue 1998, p. 19-27.

CARVALHO, Maria Carmen Romcy de. Bibliotecas universitárias brasileiras e a implantação de repositórios institucionais. Revista informação e universidade. v.1, n. 0, jul. 2009. Disponível em: < www.siglinux.nce.ufrj.br/.../artigomcarmen1-200911pdf> Acesso em: 5 nov. 2012.

HALBWACHS, Maurice. A memória coletiva. São Paulo: Centauro, 2006.

LAMBERT, M. B. M.A. O novo papel do profissional da informação na sociedade da informação. Ciência da Informação, Brasília, v. 35, n. 3, p. 125-135, set/nov. 2000.

INSTITUTO de física-UFRJ 45 anos. Rio de Janeiro: Instituto de Física, 2010. 56 p. 
LE GOFF, Jacques. Memória. In: Enciclopédia Einaudi. Lisboa: Imprensa Nacional/Casa da Moeda, 1990.

LÉVY, Pierre. Cibercultura. Rio de Janeiro: 34, 1999.

LEVACOV, Marília. Bibliotecas virtuais: (r)evolução? Ciência da Informação, Brasília, v.26, n.2, p. 125-135, maio/ago. 1997.

LIMA, Diana.O que se pode designar como Museu Virtual segundo os museus que assim se apresentam. In: ENCONTRO NACIONAL DE PESQUISA E PÓS-GRADUAÇÃO EM CIÊNCIA DA INFORMAÇÃO, 10, 2009, João Pessoa. Anais... João Pessoa: UFPB, ANCIB. 2009. Disponível em: <http://dci2.ccsa.ufpb.br:8080/jspui/handle/123456789/531>

ROBERT, Jean-Claude. Les rapports entre I'histoire e I'archivistique. In: - La place de l'archivistique dans la gestion de l'information: perspectives de recherche. Montreal: Université de Montreal, 1990.

SCHWEIBENZ, Werner. O desenvolvimento dos museus virtuais. Icom News (Newsletter of the International Council of Museums) dedicated to Virtual Museums, v. 57, n. 3, 2004, p. 3.

SCHEINER, T. Museologia, Identidade, Desenvolvimento Sustentável: estratégias discursivas. In: ENCONTRO REGIONAL DO ICOFOM, 9, 2000, Rio de Janeiro. Anais...Rio de Janeiro:2000.

SILVEIRA, Carlos Eduardo Ribeiro. Fragmentos Urbanos: o patrimônio e a construção das paisagens simbólicas nas cidades contemporâneas. 2009. Dissertação (Mestrado). Programa de Pós-Graduação em Museologia e Patrimônio,UNIRIO/MAST, Rio de Janeiro, 2009. 86f.

UFRJ-história. Disponível em:

$<$ http://www.ufrj.br/pr/conteudo pr.php?sigla =HISTORIA $>$. Acesso em:

13 mar. 2012.

UNESCO - United Nations Educational, Scientific, and Cultural Organization. Charter on the Preservation of Digital Heritage. Paris, 2003. Disponível em: <http://www.unesco.org/new/en/communication-andinformation/resources/official-documents/> Acesso em: 08 set. 2012. 